4. Suh G. S et al. Nature 431, 854-859 (2004)

5. Yu, D. etal Cell123, 545-557(2005).

6. Heisenberg, M.\& Wolf, R. J.Comp.Physiol. A 130, 113-130 (1979)

7. Dill,M etal. Nature 365,751-753 (1993)
8. Sziber, P. P.et a. Cell 37, 205-215 (1984).

9. Zars, T.etal. Science 288, 672-675 (2000)

10. Hanesch U, Fischbach, K. F. \& Heisenberg M. Cell Tissere Res 257, 343-366 (1989)

i1. Lee, T. \& L Luo, L. Trends Neurosd. 24, 251-254 (2001).

\title{
CHEMISTRY
}

\section{Catalysts live and up close}

\author{
Bert M. Weckhuysen
}

\section{Designing efficient solid-state catalysts would be easier if we knew which parts of them do what. Fluorescence microscopy could help: the technique allows single catalytic events to be observed in real time.}

Catalysts are the workhorses of the chemical industry: more than $80 \%$ of all modern chemicals come into contact with at least one catalyst during their manufacture. They can also be extremely complex: solids with large surface areas, for example, possess many potential active sites in their crystal structure. In this issue, Roeffaers et al. ${ }^{1}$ (page 572 ) première a method of 'filming' single catalytic events in real time on a solid crystal, allowing catalytic activity to be mapped over its whole surface. The innovation is a step further on the road to the 'rational design' of catalysts, which offers the prospect of improved formulations for existing catalysts, and more effective and selective catalysts created from scratch.

Rational design remains in most cases a pipe-dream: the experimental tools available for monitoring catalysts in action are still, in the main, too rudimentary. Nevertheless, this area of research - often referred to as in situ spectroscopy - has seen tremendous progress over the past decade, partially as a result of improvements in analytical instrumentation ${ }^{2-6}$. The latest contribution ${ }^{1}$ exploits a method known as fluorescence microscopy. This technique has been used, for instance, in combinatorial catalysis, to screen whole series of catalyst libraries for the formation of fluorescence, and for monitoring the disappearance of specific organic molecules during catalytic transformation $\mathrm{s}^{7-9}$. It has also been used to track the diffusion patterns of fluorescent molecules, and to determine their diffusion coefficients in different porous oxides ${ }^{10,11}$. Furthermore, fluorescence microscopy can monitor the adsorption and desorption of a dye molecule by individual crystals of a catalyst material $^{12}$.

The distinctive aspect of Roeffaers and colleagues' work ${ }^{1}$, however, is the use of highresolution fluorescence microscopy to observe a catalytic crystal at work in liquid-phase reactions. The high sensitivity of their approach enabled the authors to monitor single catalytic events in real time by observing a 'reporter' molecule that becomes fluorescent only after catalytic action. They could thus beautifully map the spatial distribution of active sites over a single catalytic crystal.
The authors tested the method on a layered double hydroxide (LDH) catalyst consisting of prismatic crystals with large basal planes and lateral faces (see Fig. 1 on page 573). Intriguingly, the tracks of the fluorescing molecules revealed that one reaction, ester hydrolysis, is catalysed only by active sites on the lateral faces of the LDH particle, whereas another reaction, transesterification, occurs on the entire outer crystal surface. In the terminology of surface scientists, ester hydrolysis is a structure-sensitive reaction, whereas transesterification is not.

Such 'crystal-face-dependent' catalysis has already been observed on metal single-crystal surfaces, for example in the synthesis of ammonia, where the rate of product formation is found to depend critically on the orientation of the crystalline iron catalyst ${ }^{13}$. But the current work is, I believe, the first of its kind to use catalytic solids in liquid-phase applications.

Whether fluorescence microscopy can be introduced as a more general method for in situ spectroscopy in catalysis research clearly depends on the availability of a sufficient range of fluorescent molecules that respond to different reactions. Attention should also be paid to the question of whether fluorescent reporter molecules behave like non-fluorescent molecules, and so indeed reproduce the distribution of catalytic sites reliably. Finally, fluorescence quenching - which may significantly affect the intensity of fluorescent emission - could become a problem, especially when the approach is extended to more severe reaction conditions.

Currently, work on the in situ spectroscopy of catalytic solids is divided, roughly speaking, into two groups, probably reflecting the differing expertise of the chemists involved. The first group focuses on the inorganic part of the catalyst material, and aims to capture its oxidation state and the geometrical structure of its bonds (its 'coordination environment'). This group uses techniques such as absorption spectroscopy and electron paramagnetic resonance. The second group, which focuses on the organic parts of the catalyst, uses nuclear magnetic resonance and vibrational spectroscopic techniques, such as infrared and Raman spectroscopy, to illuminate reaction mechanisms and potential reaction intermediates.

With Roeffaers and colleagues' work ${ }^{1}$, the organic chemists gain a new tool for their in situ spectroscopic work. Further efforts should be directed towards combining both schools of thought, focusing, with nanometre resolution, on both the inorganic and organic parts of a catalyst. That could allow crucial insights into changes in the coordination environment of an active site, as well the reaction intermediates formed. The results could then be interpreted in terms of catalytic reactivity and selectivity in a 'joined-up' fashion. Although combining two or more spectroscopic techniques might seem simple, many hurdles must be cleared before it becomes a reality ${ }^{14,15}$.

But the main challenge is to push the resolution of in situ spectroscopic tools into the nanometre range. Transmission electron microscopy offers resolution at the atomic scale (around a tenth of a nanometre). Coupling this with electron energy-loss spectroscopy, in which the sample is bombarded with a monoenergetic beam of electrons, allows chemical mapping of a catalytic surface. However, the energy resolution of this technique is still too low to identify the different oxidation states of the bombarded material. Moreover, electronbased techniques also need low pressures (in the millibar range), and thus the results obtained may deviate from those of experiments performed under true reaction pressures.

So there is room for other spectroscopic tools that, in combination with Roeffaers and colleagues' fluorescence microscopy ${ }^{1}$, could deliver both inorganic and organic information about a catalytic process, creating a powerful in situ nanospectroscopy technique for taking snapshots of a wide variety of catalytic solids at work. If this endeavour succeeds, rational catalyst design will be a step closer to realization.

BertM. Weckhuysen is in the Department of Chemistry, Debye Institute, Utrecht University,

Sorbonnelaan 16, PO Box 80083, 3508 TB

Utrecht, the Netherlands.

e-mail:b.m.weckhuysen@chem.uu.nl

1 Roeffaers, M. B. I et al. Nature 439,572-575(2006)

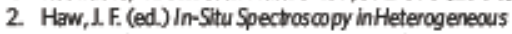
Catalysis(Wiley-VCH, Weinheim, 2002).

3 Weckhuysen, B. M. (ed) In-Situ Spectroscopyof Catalyst (Am.Sci, Stevenson Ranch, CA, 2004)

4. Weckhuysen, B. M. Chem. Commun 97-110 (2002).

5. Hunger, $M$ \& Weitkam 1 LAngew. Chem. Int. Edn 40 , 2954-2971 (2001).

6. Thomas, 1. M. Angew. Chem. hit. Edh 38,3589-3628 (1999)

7. Shaughnessy, K. H. Kim, P.\&. Hartwig, J.F. J. Am. Chem. Soc. 121, 2123-2132(1999)

8. Copeland, G.T.\& MillerS. I.A.Am. Chem.Soc. 121, 4306-4307 (1999).

9. Su, H.et al. Anal. Chem. 73,4434-4440 (2001).

10. Helliriegel, C. etal. N.J.Phys. 7, 23(2005).

11. Seebacher, C et al. J.Phys Chem. B 106, $5991-5995$ (2001).

12. Roeffaers, M. B.l et al. ChemPhysChem 6, 2295-2299 (2005)

13. Spencer, N.D. et al. J. Catal. 74, 129-135 (1982)

14. Bruckner, A.Chem. Commun. 1761-1763(2005).

15. Beale, A.M. etal Chem. Commun. 3015-3017 (2005) 